\title{
Reprodução experimental da doença articular degenerativa, pelo método cirúrgico associado à terapia condroprotetora, em ratos
}

\section{Chondroprotectant therapy in rats with degenerative joint disease experimentally transected cranial cruciate}

\author{
Marcos Marini Melo ${ }^{1 *}$; Paulo Ricardo Mallmann²; Dábila Aráujo Sonego ${ }^{2}$; \\ Alessandro Tadeu Marques ${ }^{3}$; Gentil Ferreira Gonçalves ${ }^{4}$; Rosana Zanatta \\ Marcos Almeida Souza ${ }^{6}$; Pedro Eduardo Brandini Néspoli ${ }^{7}$; Roberto Lopes Souza ${ }^{8}$
}

\section{Resumo}

\begin{abstract}
A osteoartrite (OA) é a doença articular mais comum em humanos e animais, o que ocasiona restrição de movimentos e dor, na região acometida. Tal enfermidade afeta mais de $25 \%$ dos humanos acima de 60 anos e, aos 70 anos, ela é considerada universal. Estima-se que nos Estados Unidos da América, 20\% ou mais da população canina é acometida pela OA. O presente estudo tem como finalidade avaliar a terapia medicamentosa (polisulfato de pentosano, betametasona e sulfato de condroitina/glucosamina), em um modelo experimental de OA cirurgicamente induzido, em ratos. A doença articular foi promovida pela transecção cirúrgica do ligamento cruzado cranial, sendo que, após o procedimento cirúrgico, os animais foram mantidos e tratados por oito semanas. Foi realizada avaliação radiográfica e tomográfica antes e oito semanas após a indução da osteoartrite. Todos os animais foram submetidos à eutanásia para análise macroscópica e microscópica, que foram realizadas para avaliar a progressão da enfermidade e a ação terapêutica. $\mathrm{Na}$ análise macroscópica foi observadas lesões nos joelhos submetidos à indução OA. Os animais não tratados apresentaram lesões graves enquanto que, os animais tratados apresentaram lesões leves a moderadas. Concluiu-se que o polissulfato de pentosano é eficiente no tratamento de lesões articulares iatrogênicas de ratos, uma vez que outros tratamentos não mostraram qualquer diferença significativa.

Palavras-chave: Osteoartrite, joelho, rato, polisulfato de pentosano, sulfato de condroitina, sulfato de glucosamina, betametasona
\end{abstract}

\footnotetext{
Abstract

Osteoarthritis (OA) is the most common joint disease in both humans and animals, and it results in movement restriction and pain at the affected area. This disorder affects more than $25 \%$ of people over 60 years of age, and it is considered universal for 70 -year-old people. OA is estimated to affect over $20 \%$ of the canine population from the United States of America. The present study aimed to evaluate

${ }^{1}$ M.e. em Ciências Veterinárias, Faculdade de Agronomia e Medicina Veterinária, UFMT, Cuiabá, MT. E-mail: melomm@terra.com.br

2 Discente(s) da Faculdade de Agronomia e Medicina Veterinária, UFMT, Cuiabá, MT. E-mail: pmallman1@gmail.com; dabila_s@ hotmail.com

${ }^{3}$ Discente de Mestrado da Faculdade de Odontologia, UNIC, Cuiabá, MT. E-mail: alessadrotadeu@krotoniuni.com.br

${ }^{4}$ Prof. Adjunto da Faculdade de Medicina Veterinária, Universidade Federal da Fronteira Sul, UFS, Realeza, PR. E-mail: gferreira@ ufmt.br

${ }^{5} \operatorname{Prof}^{\mathrm{a}} \mathrm{Dr}^{\mathrm{a}}$. da Faculdade de Medicina Veterinária, Universidade de Cuiabá, UNIC, Cuiabá, MT. E-mail: rosana.zanatta@gmail.com

${ }^{6}$ Prof. do Dept ${ }^{\circ}$ de Clinica Médica Veterinária, UFMT, Cuiabá, MT. E-mail: souzavet@gmail.com

${ }^{7}$ Prof. Dr. em diagnóstico por imagem veterinária, Dept ${ }^{\circ}$ de Clínica Médica Veterinária, UFMT, Cuiabá, MT. E-mail: nespolipb@ gmail.com

${ }^{8}$ Prof. Dr. do Programa de Pós Graduação em Ciências Veterinárias, UFMT, Cuiabá, MT. E-mail: rsouza@ufmt.br

* Autor para correspondência
} 
the treatment of rats that were surgically induced to OA using two different drug therapies, one with pentosan polysulfate, one with betamethasone, and one with chondroitin sulfate/ glucosamine. In order to produce joint disease, the cranial cruciate ligament was surgically transected. Animals were kept and treated for eight days after surgery and were assessed via both radiographies and tomographies taken before surgery and eight weeks later. All animals were euthanized having both macroscopic and microscopic analysis performed to evaluate the disorder progression and therapeutic action. Macroscopic analysis showed lesion in the knees subjected to OA induction. The untreated animals presented major lesions whereas the treated ones presented mild to moderate lesions. In conclusion, pentosan polysulfate is recommendable for the treatment of iatrogenic joint lesions in rats since the other treatments showed no significant difference.

Key words: Osteoarthritis, knee, rat, pentosan polysulfate, chondroitin sulfate, chondroitin sulfate, glucosamine, betamethasone

\section{Introdução}

A osteoartrite (OA) é uma doença articular degenerativa (DAD) progressiva, também conhecida como osteoartrose. Apresenta alta prevalência em humanos e animais, especialmente, caninos, equinos e suínos, sendo considerada uma das mais comuns nestas espécies (BROOKS, 2002; BIASI et al., 2005; SCOTT, 2007). OA é um processo não inflamatório, não infeccioso das estruturas articulares, principalmente da cartilagem articular (SCHAWARZ, 2008). As lesões iniciamse pela perda da superfície regular e lisa da cartilagem articular, podendo se estender até aos processos reparativos e/ou formação de osteófitos (TAKIZAWA et al., 2008).

A terapia da OA consiste em retardar ou diminuir a evolução das lesões na região articular (INNES; BARR; SHARIF, 2000), podendo requerer uma abordagem medicamentosa e/ou cirúrgica. A terapia medicamentosa inclui a associação de fármacos, como anti-inflamatórios não esteroidais (AINES), glicocorticóides, analgésicos, nutracêuticos e drogas modificadoras da osteoartrite (DMOAs) (CALAMIA et al., 2011). A terapia cirúrgica envolve principalmente a curetagem da superfície articular e estabilização da articulação por restauração da ruptura do ligamento cruzado cranial (RLCCr), que é uma das principais causas da OA (FERREIRA et al., 2009).

A etiopatogenia da OA articular é extremamente complexa, sendo seu diagnóstico clínico e laboratorial geralmente realizado em um estágio avançado, tornando o tratamento menos efetivo. Este estudo experimental visa estabelecer a relação entre a progressão e a terapia medicamentosa relacionados a $\mathrm{OA}$, principalmente no início da lesão e do uso de alguns fármacos, tanto de ação anti-inflamatória quanto nutracêutica, perante avaliação por radiografia convencional e tomografia "cone beam".

\section{Metodologia}

\section{Animais}

Este experimento foi executado nas dependências do Laboratório Experimental de Cirurgia e do Laboratório Patologia Veterinário da Universidade Federal de Mato Grosso. O estudo experimental foi aprovado pelo comitê de ética ${ }^{\circ} 23108.002089 / 11$ 8.

Para esta pesquisa, foram utilizados 20 ratos Wistar machos, provenientes do Biotério Central da UFMT. Os animais foram padronizados com o peso entre 220 e $240 \mathrm{~g}$ e idade de três meses. Antes do inicio do experimento os animais ficaram em gaiolas individuais, à temperatura constante de 22 $\pm 2{ }^{\circ} \mathrm{C}$, com ciclo claro/escuro de 12 horas durante duas semanas. Água e ração foram fornecidas " $a d$ libitum".

Após o período de adaptação, realizou-se o sorteio que separou os animais em quatro grupos distintos e cada grupo com $n=5$. 
Grupo Sulfato de Condroitina e Glucosamina: os animais foram submetidos a transecção do ligamento cruzado cranial (TLCCr) e posteriormente tratados com sulfato de condroitina e glucosamina $500 \mathrm{mg} / \mathrm{kg}$ pela via subcutânea (SC) com intervalo de 72 horas durante oito semanas após cirurgia.

Grupo Polisulfato de Pentosano: os animais foram submetidos a TLCCr e posteriormente tratados com polisulfato de pentosano $3 \mathrm{mg} / \mathrm{kg}$, pela via SC com intervalo de 72 horas durante oito semanas após cirurgia.

Grupo Betametasona: animais submetidos a TLCCr e posteriormente tratados com $25 \mathrm{mg} / \mathrm{kg}$ pela via intra-articular (IA) com intervalo de 72 horas durante oito semanas após cirurgia.

Grupo Controle: animais submetidos a TLCCr e posteriormente tratados com Solução Salina $0,3 \mathrm{~mL} /$ animal pela via subcutânea com intervalo de 72 horas durante oito semanas após cirurgia.

\section{Indução OA e anestesia}

A TLCCr foi baseada no modelo Pond e Nuki (1973). No pós operatório os ratos foram medicados com $5 \mathrm{mg} / \mathrm{kg}$ de enrofloxacina pela via SC e com cloridrato de morfina $3 \mathrm{mg} / \mathrm{kg}$ pela via $\mathrm{SC}$ com intervalo de 12 horas, durante três dias. Os animais foram submetidos à anestesia com Cetamina $(60 \mathrm{mg} /$ $\mathrm{kg})$ e Xilazina $(3 \mathrm{mg} / \mathrm{kg})$ administradas pela via intraperitoneal (IP) (STEFEY, 1996).

\section{Avaliação radiográfica}

Foi realizado o exame radiográfico no período de seleção dos animais e após o tratamento. O exame radiográfico com o uso de aparelho de radiografia convencional Raicon SH 300D ${ }^{\circledR}$ e filme de mamografia AD-M Fujifilm ${ }^{\circledR}$. Cabe salientar que houve necessidade de anestesiar os animais com o mesmo protocolo anestésico realizado na indução da OA, sendo que os mesmos encontravam-se em decúbito dorsal na mesa radiográfica. As radiografias foram efetuadas em duas projeções (ventro-dorsal e latero-lateral) da articulação do joelho. A técnica utilizada nos exames foi de 100MA, $67 \mathrm{Kv}$ e 8 Mas. Todos os animais selecionados para o estudo possuíam lesões com escore 0 segundo Torelli et al. (2004).

\section{Avaliação tomográfica}

O exame tomográfico foi realizado no aparelho Kodak 9000 3D® no período de adaptação e após os tratamentos. Previamente à tomografia, os animais foram submetidos à anestesia com o protocolo anestésico já mencionado, e dispostos em decúbito dorsal, sobre uma calha de vidro tendo o membro pélvico esquerdo estendido e fixado na haste do tomógrafo. Após o posicionamento dos animais realizou-se a calibragem do tomógrafo, seguido da execução da tomografia do joelho acometido, que permitiu a captura das imagens.

As imagens foram geradas com a utilização do programa Kodak Dental Imaging software 3D module $2,4 \AA$ e o emprego do protocolo de $63 \mathrm{KV}$, $2,50 \mathrm{~mA}, 10,80 \mathrm{~s}$ e $46 \mathrm{mGy} \cdot \mathrm{cm}^{2}$. As tomografias foram avaliadas sob o mesmo critério da avaliação radiográfica, no modelo de Torelli et al. (2004). O corte da avaliação foi de $0,76 \mu \mathrm{m}$ na secção oblíqua, o qual se permite visualizar a peça em $360^{\circ}$ nos planos horizontal e vertical.

Tais avaliações foram por meio de estudo cego e simples, que utilizaram como parâmetro a classificação de Torelli et al. (2004), onde se verificou a presença e a intensidade das lesões no osso subcondral e na superfície articular (osteófitos e osteólise), com base em sua estrutura anatômica de compartimentos: côndilo femoral medial, côndilo medial lateral, platô tibial lateral, platô tibial medial e tróclea, obedecendo a escala de 0 a 3 . Cada joelho poderia obter a pontuação máxima de 30 pontos (Tabela 1). 
Tabela1. Níveis de escores crescentes e critério de uso nas avaliações radiográficas e tomográficas dos joelhos submetidos a TLCCr.

\begin{tabular}{lll}
\hline & \multicolumn{1}{c}{ ESCORE } & LESÃO \\
\hline 0 & Ausente & Sem lesão \\
1 & Leve & Lesão discreta, localizada e menos grave. \\
2 & Moderado & Lesão generalizada, mas moderadamente grave. \\
3 & Grave & Lesão generalizada e muito grave. \\
\hline
\end{tabular}

Fonte: Torelli et al. (2004).

\section{Análise estatística}

O experimento seguiu um delineamento inteiramente casualizado com cinco repetições em que os tratamentos se constituíam na aplicação dos seguintes medicamentos: betametasona, sulfato de condroitina e glucosamina, polisulfato de pentosano.

As análises estatística foram obtidas por meio do software $R^{\circledR}$ (2012), que utilizou o teste $F$, para verificar a diferença entre os tratamentos e o teste de Scott-Knott para comparação de médias. Para verificar as pressuposições do teste $\mathrm{F}$, foi realizado o teste de Shapiro-wilk para normalidade dos erros e do teste de Levene, foi adotado o nível de significância de 5\%.

\section{Resultados e Discussão}

Os achados radiográficos restringiram-se na osteólise nos côndilos medial e lateral (escore 2) e com menor intensidade (escore 1) no platô tibilal medial e lateral (Figura 1).

O grupo tratado com polisulfato de pentosano foi o que menos apresentou lesões. Já os outros grupos não apresentaram diferença significativa quando comparado com o grupo controle. O desempenho terapêutico perante a avaliação radiográfica é apresentada na Figura 2.

Nas avaliações tomográficas "cone beam" verificaram-se as mesmas lesões descritas nos achados radiográficos e também a presença de osteófitos (escore 1) conforme figura 3.

Como na avaliação radiográfica, o grupo tratado com polisulfato de pentosano foi o que apresentou a menor intensidade de lesões. Os grupos medicados com sulfato de condroitina e glucosamina e betametasona não diferiram de forma significativa (Figura 4).

$\mathrm{Na}$ avaliação de pequenas estruturas como o joelho de ratos, a tomografia "cone beam" demonstrou ser uma ferramenta de diagnóstico por imagem de maior precisão, quando comparada a radiografia convencional (Figura 5).

$\mathrm{Na}$ literatura a maioria dos estudos de OA realizaram RX e TM em conjunto. Mcerlain et al. (2008) induziram OA em ratos pelo método cirúrgico e avaliaram a evolução da doença articular degenerativa durante oito semanas, apenas pela micro-tomografia. Estes mesmos autores descreveram que $92 \%$ dos ratos estudados apresentaram lesões radioluscentes nos côndilos femorais e no platô tibial e $33 \%$ dos ratos manifestaram calcificação do tendão patelar. Os resultados aqui apresentados não diferiram dos encontrados por Mcerlain et al. (2008), exceto a calcificação do tendão patelar.

Torelli et al. (2004) desenvolveram um estudo que induziu a OA em coelhos, por imobilização do membro pélvico, durante 12 semanas. Os animais foram avaliados por exames de raio-x, microtomografia e ressonância magnética, em períodos diferentes. Os achados radiográficos consistiram em: diminuição do espaço intra-articular, osteólise no osso subcondral (fêmur e tíbia) e calcificação do tendão patelar. Enquanto isso, os achados da microtomografia foram: osteólise no osso subcondral (fêmur e tibia), ligeira diminuição do espaço intraarticular e osteófitos marginais. 
Figura 1. Na imagem A observa-se articulação do joelho de rato saudável. As imagens B e C representam o grupo tratado com polisulfato de pentosano. As Imagens $\mathrm{D}$ e $\mathrm{E}$ correspondem aos animais medicados com sulfato de condroitina e glucosamina. As imagens $\mathrm{F}$ e $\mathrm{G}$ pertencem ao grupo medicado com betametasona. A imagem $\mathrm{H}$ pertence ao grupo controle. As setas indicam lise da cartilagem articular e esclerose do osso sub-condral.

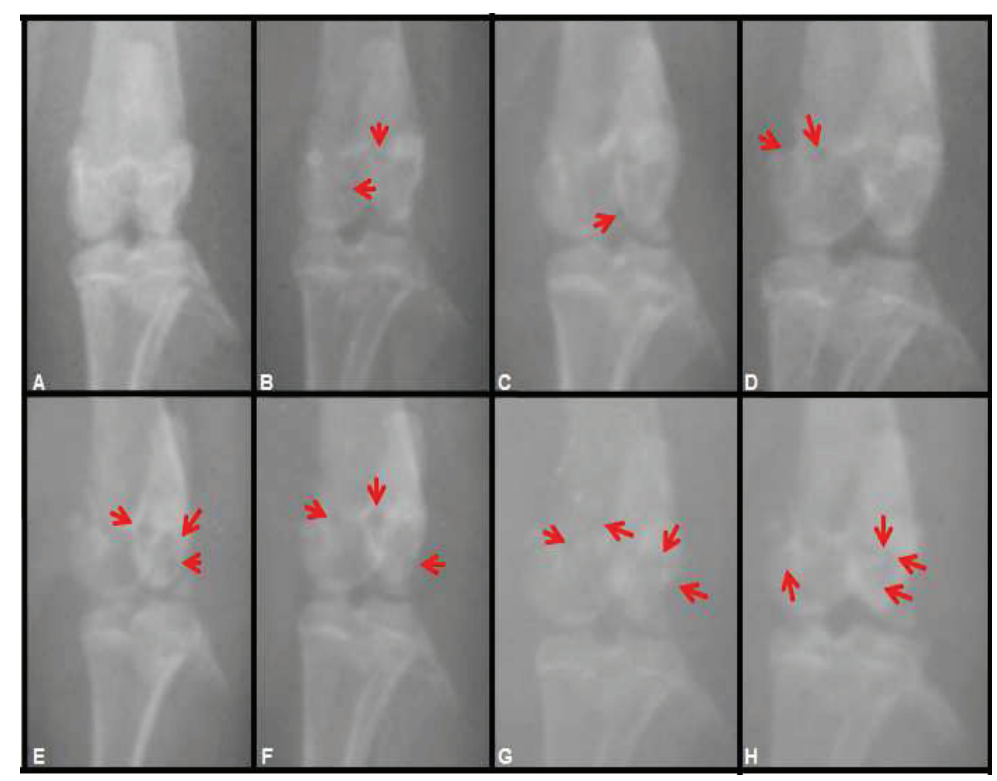

Fonte: Elaboração dos autores.

Figura 2. A intensidade de lesão obtida na avaliação radiográfica em joelhos acometidos com OA segundo a classificação de Torelli et al. (2004). Letras diferentes se diferem estatisticamente ao nível de $p<0,05$.

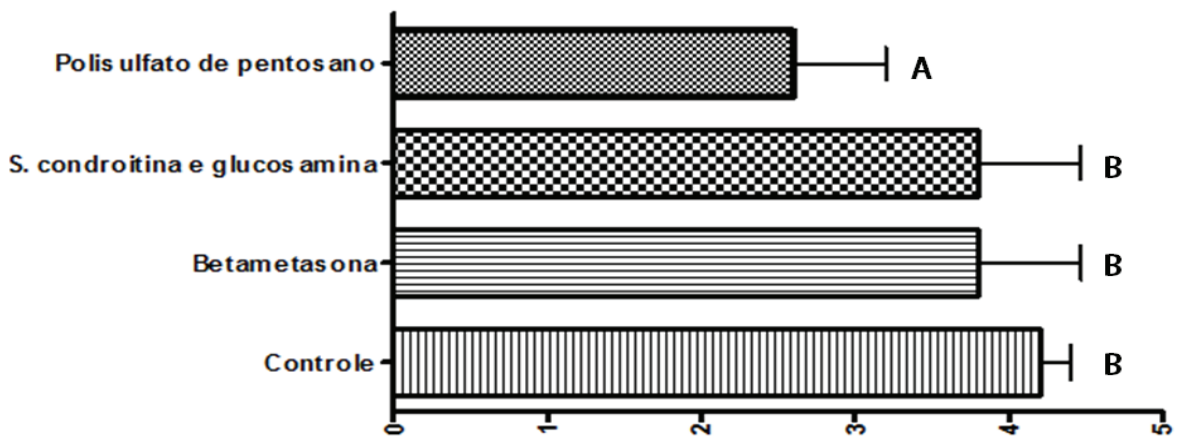

Fonte: Elaboração dos autores. 
Figura 3. A imagem A demonstra uma tomografia "cone beam" no perfil lateral da articulação do joelho normal. Na imagem B observa-se osteólise no côndilo. Na imagem C (ver setas) observa-se osteólise no côndilo femoral e no platô tibial, além de apresentar osteófito entre o côndilo e platô tibial. Na imagem D e E observam-se osteólise no côndilo femoral. Na imagem F observa-se esteólise femoral e osteófito femoral.

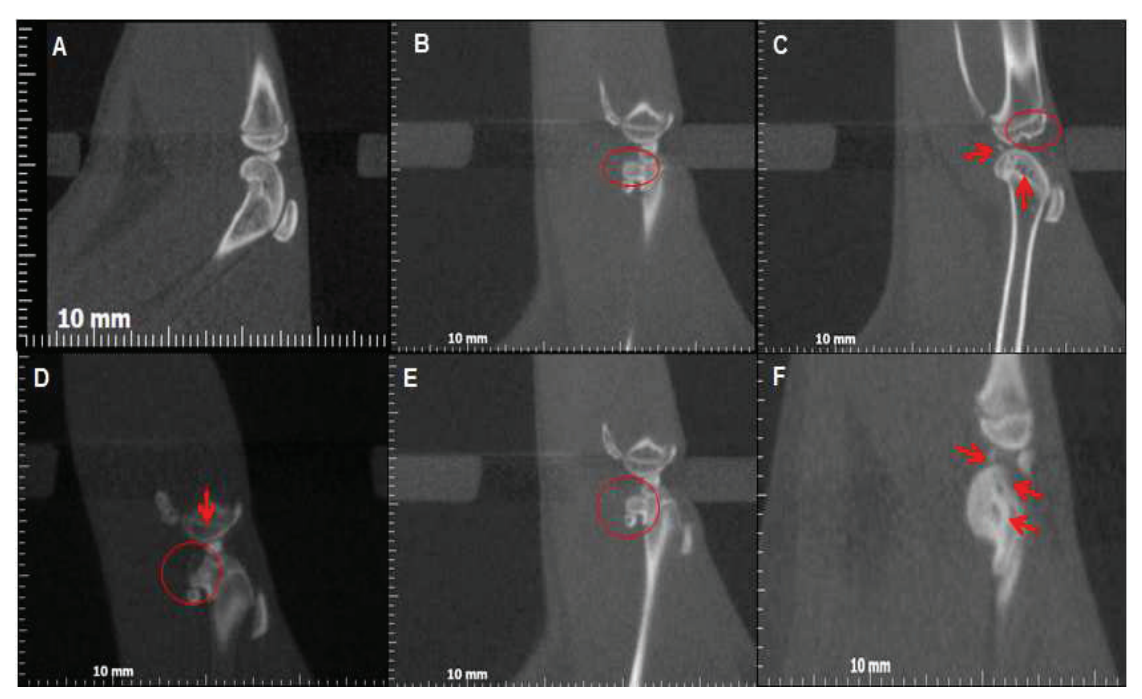

Fonte: Elaboração dos autores.

Figura 4. A intensidade de lesão obtida na avaliação tomográfica em joelhos acometidos com OA segundo a classificação de Torelli et al. (2004). Letras diferentes se diferem estatisticamente ao nível de p<0,05.

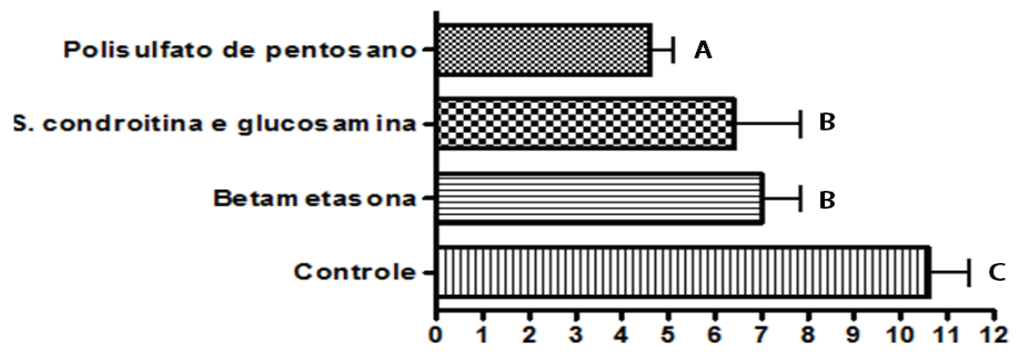

Fonte: Elaboração dos autores. 
Figura 5. Comparação da lesão por meio RX e TM em distintos grupos terapêuticos.

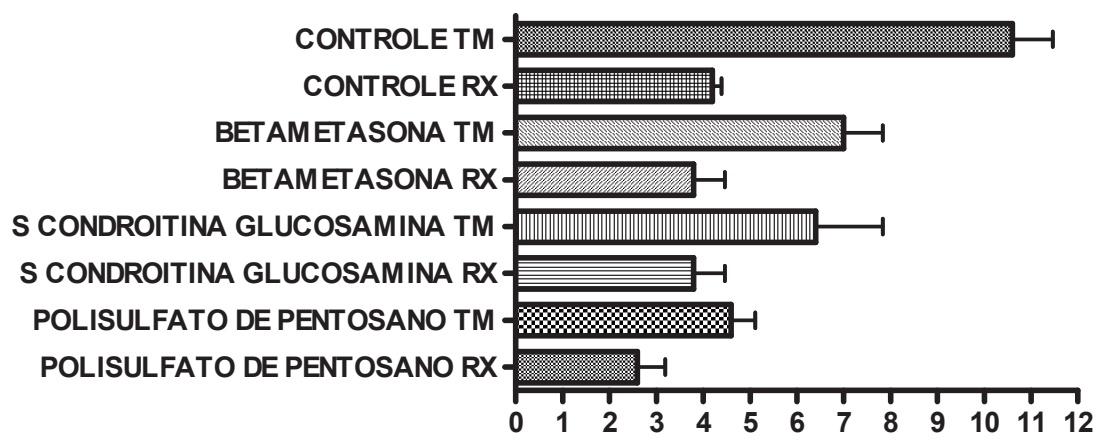

Fonte: Elaboração dos autores.

O protocolo terapêutico com polisulfato de pentosano inibiu a progressão da DAD. O polisulfato de pentosano é uma molécula semelhante à heparina e tem-se atribuído a esta substância o aumento da vascularização do osso subcondral e conseqüentemente, o aporte de nutrientes à cartilagem acometida pela $\mathrm{DAD}$, o que favorece o processo de cicatrização segundo sugeriram Budsberg et al. (2007). Como no estudo descrito por Ghosh (1999) onde o polisulfato de pentosano abrandou de maneira significante a evolução da OA, provavelmente, os resultados favoráveis a este fármaco se devem às suas propriedades antitrombótica, anti-inflamatória e ação fibrinolítica, o que melhoraria o aporte sanguíneo para o osso subcondral trabecular.

Estudos sobre o plasma de cães com OA têm demonstrado que eles têm uma capacidade reduzida para dissolver coágulos de fibrina em comparação com o plasma de cães não-osteoartríticas. Este defeito na fibrinólise, medido como tempo de lise do coágulo de euglobulina, contribui para a trombose do osso subcondral periarticular e que podem produzir dor e osteonecrose nas articulações afetadas. Tratamento com polisulfato de pentosano em quatro injeções $(3 \mathrm{mg} / \mathrm{kg})$ em intervalos semanais normalizaram o tempo de lise do coágulo de euglobulina em cães OA. Este efeito foi ainda evidente quatro semanas após a última injeção. (GHOSH; CHERAS, 2001).
Rogachefsky et al. (1993) induziram experimentalmente a OA em caninos pelo modelo Pond e Nuki (1973). Três semanas após a indução, iniciou-se o tratamento com polisulfato de pentosano na dose de $2 \mathrm{mg} / \mathrm{kg}$ durante três semanas. Após o tratamento procedeu-se com avaliação macroscópica da articulação do joelho, que não houve presença de lesão significativa, quando comparada com o grupo controle. Posteriormente houve análise histológica, onde se verificou que o nível de lesão na cartilagem do grupo tratado foi expressivamente inferior ao do grupo controle. Além disso, constatou-se queda brusca no nível de metaloproteinase no grupo medicado com polisulfato de pentosano, quando comparado com o grupo controle.

Mcilwraith, Frisbie e Kawcak (2012) induziram OA no carpo de equinos, onde se observou que, naqueles animais tratados com polisulfato de pentosano, houve menor intensidade de claudicação quando comparado com os outros animais. Cabe ainda ressaltar que, os achados microscópicos como a fibrilação da cartilagem articular foi substancialmente reduzida devido ao tratamento com polisulfato de pentosano.

Segundo Papich (2009), o uso de o sulfato de condroitina e glucosamina tem sua eficácia duvidosa na terapêutica da OA. Em humanos, o sulfato de condroitina e glucosamina também tem seu uso questionado, principalmente no que se refere à 
dosagem e via de administração (CALAMIA et al., 2011).

No estudo experimental conduzido por Laverty et al. (2005), realizado em éguas tratadas com glucosamina, verificou-se o aumento significativo do nível sérico de glucosamina no líquido sinovial e soro sanguíneo até 12 horas após a administração medicamentosa. Segundo o estudo realizado por Volpi (2002), em humanos sadios que foram medicados com sulfato de condroitina bovina na dose de $1500 \mathrm{mg}$, apresentou o pico sérico plasmático em duas horas após a administração do medicamento, porém a sua biodisponibilidade perdurou somente por quatro horas. $\mathrm{O}$ mesmo pesquisador repetiu o estudo em humanos sadios medicados com sulfato de condroitina oriunda de molusco na dose de $1500 \mathrm{mg}$, onde revelou o mesmo tempo para atingir o pico sérico plasmático. No entanto, diferenciouse no tempo de biodisponibilidade que chegou a aproximadamente nove horas (VOLPI, 2003). Já o estudo realizado por Persiani et al. (2005) em humanos sadios medicados com sulfato de condroitina e glucosamina detectou absorção de $90 \%$, porém a biodisponibilidade do sulfato de condroitina foi de $56 \%$, sendo esta indesejada para o tratamento de OA.

Com relação à betametasona intra-articular, esta inibe o processo inflamatório intra-articular, o que confere a analgesia secundária pela sua ação anti-inflamatória (ALBANO et al., 2009). $\mathrm{Na}$ medicina humana, no trabalho conduzido por Hetland et al. (2006), os pacientes acometidos pela doença articular degenerativa foram tratados com doses semanais de betametasona intra-articular por 52 semanas, onde se verificou posteriormente rápida melhora clínica e retardo na progressão da enfermidade. Em contrapartida, Horisawa et al. (2002) afirmam que a infiltração intra-articular em joelhos de coelhos com OA ocasiona como efeito indesejado, a inibição de mitose e depleção dos condrócitos. Além do fato que, Habib e Safia (2009) alertam para o uso cauteloso em diabéticos, pelo risco de hiperglicemia.

\section{Conclusão}

Neste estudo, a tomografia possibilitou melhor avaliação das articulações acometidas por OA uma vez que, os joelhos avaliados obtiveram uma pontuação maior quando comparada com a avaliação radiográfica.

Entre os tratamentos utilizados neste estudo, o polisulfato de pentosano se mostrou com maior eficácia no impedimento da progressão da lesão, quando comparado com os tratamentos realizados. Portanto, pode-se afirmar que o polisulfato de pentosano demonstrou ser uma opção terapêutica promissora no tratamento das osteoartrites.

\section{Referências}

ALBANO, M. B.; SKROCH, G. P.; IOSHII, S. O.; GRAHELS, X. S.; ALENCAR, P. G.; MATIAS, J. E.; Computerized photocolorimetric analysis of the effects of intraarticular betamethasone on the proteoglycan concentration of leporine knee cartilage matrix: influence of the number of intraarticular injections. Revista do Colégio Brasileiro de Cirurgiões, v. 36, n. 3, p. 256-60, jul. 2009. Disponível em: <http://www.ncbi.nlm.nih.gov/ pubmed/20076907>. Acesso em: 14 mar. 2011.

BIASI, F.; RAHAL, S. C.; VOLPI, R. S.; SEQUEIRA, J. L. Cranial cruciate ligament reconstruction in dogs associated or not to chondrointin sulfate. Arquivo Brasileiro Medicina Veterinária Zootecnia, v. 57, n. 4 , p. $442-447,2005$. Disponível em: < http:// www.scielo.br/scielo.php?script=sci_arttext\&pid $=$ S0102-09352005000400004>. Acesso em: 10 jan.2010.

BROOKS, P. Impact of osteoarthritis on individuals and society: how much disability? Social consequences and health economic implications. Current Opinion in Rheumatology, v. 14, n. 5, p. 573-7, sep. 2002. Disponível em: <http://www.ncbi.nlm.nih.gov/entrez/ query.fegi? $\mathrm{cmd}=$ Retrieve $\& \mathrm{db}=$ PubMed $\&$ dopt $=$ Citation \&list_uids $=12192258>$. Acesso em: 10 out. 2010.

BUDSBERG, S. C.; BERGH, M. S.; REYNOLDS, L. R.; STREPPA, H. K. Evaluation of pentosan polysulfate sodium in the postoperative recovery from cranial cruciate injury in dogs: a randomized, placebo-controlled clinical trial. Veterinary Surgery, v. 36, n. 3, p. 234-44, apr. 2007. Disponível em: <http://www.ncbi.nlm.nih. gov/pubmed/17461948>. Acesso em: 10 jan. 2010. 
CALAMIA, V.; FERNÁNDEZ, P.; MATEOS, J.; LOURIDO, L.; ROCHA, B.; MONTELL, E.; VERGÉS, J.; RUIZ, C.; BLANCO, F. J. Pharmacoproteomic study of three different chondroitin sulfate compounds on intracellular and extracellular human chondrocyte proteomes. Molecular \& Cellular Proteomics, v. 11, n. 11, p. 1097-1104, dec. 2011. Disponível em: <http:// www.ncbi.nlm.nih.gov/pubmed/22203690>. Acesso em: 14 maio 2012.

FERREIRA, M.; SCHANAIDER, A.; SILVA, P. C.; ABREU, A. V.; COSTA, A. F.; BRAGA, J. M.; ROUSSO, F. R.; PEREIRA, L. P. Study of the extra articulate syndesmoplasty technique with autogenous fascia lata. A model in dogs. Revista do Colégio Brasileiro de Cirurgiões, v. 36, n. 2, p. 161-6, apr. 2009. Disponível em: $<$ http://www.ncbi.nlm.nih.gov/entrez/query.fcgi $? \mathrm{cmd}=$ Retrieve $\& \mathrm{db}=$ PubMed $\&$ dopt $=$ Citation\&list_ uids $=20076889>$. Acesso em: 10 jan.2010.

GHOSH, P. The pathobiology of osteoarthritis and the rationale for the use of pentosan polysulfate for its treatment. Seminars in Arthritis and Rheumatism, v. 28, n. 4, p. 211-67, feb. 1999. Disponível em: <http://www. ncbi.nlm.nih.gov/pubmed/10073500>. Acesso em: 14 jun. 2010.

GHOSH, P.; CHERAS, P. A. Vascular mechanisms in osteoarthritis. Best Practice \& Research Clinical Rheumatology, v. 15, n. 5, p. 693-709, dec. 2001. Disponível em: <http://www.ncbi.nlm.nih.gov/pubmed/ 11812016>. Acesso em: 14 jun, 2010.

HABIB, G.; SAFIA, A. The effect of intra-articular injection of betamethasone acetate/betamethasone sodium phosphate on blood glucose levels in controlled diabetic patients with symptomatic osteoarthritis of the knee. Clinical Rheumatology, v. 28, n. 1, p. 85-87, jan. 2009. Disponível em: <http://link.springer.com/article/ 10.1007\%2Fs10067-008-1023-9>. Acesso em: 10 jan. 2010.

HETLAND, M. L.; STENGAARD-PEDERSEN, K.; JUNKER, P.; LOTTENBURGER, T.; ELLINGSEN, T.; ANDERSEN, L. S.; HANSEN, I.; SKJØDT, H.; PEDERSEN, J. K.; LAURIDSEN, U. B.; SVENDSEN, A.; TARP, U.; PØDENPHANT, J.; HANSEN, G.; LINDEGAARD, H.; CARVALHO, A.; ØSTERGAARD, M.; HØRSLEV-PETERSEN, K. Combination treatment with methotrexate, cyclosporine, and intraarticular betamethasone compared with methotrexate and intraarticular betamethasone in early active rheumatoid arthritis: an investigator-initiated, multicenter, randomized, double-blind, parallel-group, placebocontrolled study. Arthritis and Rheumatism, v. 54, n. 5, p. 1401-9, may 2006. Disponível em: <http://www.ncbi. nlm.nih.gov/pubmed/16645967>. Acesso em: 10 jan. 2010.

HORISAWA, E.; HIROTA, T.; KAWAZOE, S.; YAMADA, J.; YAMAMOTO, H.; TAKEUCHI, H.; KAWASHIMA, Y. Prolonged anti-inflammatory action of DL-lactide/glycolide copolymer nanospheres containing betamethasone sodium phosphate for an intra-articular delivery system in antigen-induced arthritic rabbit. Pharmaceutical Research, v. 19, n. 4, p. 403-10, apr. 2002. Disponível em: <http://www.ncbi.nlm.nih.gov/ pubmed/12033371>. Acesso em: 10 jan. 2010.

INNES, J.; BARR, A.; SHARIF, M. Efficacy of oral calcium pentosan polysulphate for the treatment of osteoarthritis of the canine stifle joint secondary to cranial cruciate ligament deficiency. Veterinary Record, v. 146, n. 15, p. 433-7, apr. 2000. Disponível em: <http:// www.ncbi.nlm.nih.gov/entrez/query.fcgi? $\mathrm{cmd}=$ Retriev $\mathrm{e} \& \mathrm{db}=$ PubMed\&dopt $=$ Citation\&list_uids $=10811265>$. Acesso em: 10 jan. 2010.

LAVERTY, S.; SANDY, J. D.; CELESTE, C.; VACHON, P.; MARIER, J. F.; PLAAS, A. H. Synovial fluid levels and serum pharmacokinetics in a large animal model following treatment with oral glucosamine at clinically relevant doses. Arthritis and Rheummatism, v. 52, n. 1, p. 181-91, jan. 2005. Disponível em: <http://www.ncbi. nlm.nih.gov/pubmed/15641100>. Acesso em: 16 jun. 2011.

MCERLAIN, D.; APPLETON, C. T.; LITCHFIELD, R. B.; PITELKA, V.; HENRY, J. L.; BERNIER, S. M.; BEIER, F.; HOLDSWORTH, D. W. Study of subchondral bone adaptations in a rodent surgical model of OA using in vivo micro-computed tomography. Osteoarthritis and Cartilage, v. 16, n. 4, p. 458-69, apr. 2008. Disponível em: $\quad<$ http://www.ncbi.nlm.nih.gov/entrez/query.fcgi $? \mathrm{cmd}=$ Retrieve $\& \mathrm{db}=$ PubMed\&dopt $=$ Citation\&list_ uids $=17900933>$. Acesso em: 03 set. 2010.

MCILWRAITH, C. W.; FRISBIE, D. D.; KAWCAK, C. E. Evaluation of intramuscularly administered sodium pentosan polysulfate for treatment of experimentally induced osteoarthritis in horses. American Journal of Veterinary Research, v. 73, n. 5, p. 628-33, may 2012. Disponível em: <http://www.ncbi.nlm.nih.gov/ pubmed/22533393>. Acesso em: 11 ago. 2012.

PAPICH, M. G. Manual saunders terapêutico veterinário. 2. ed. São Paulo: MedVet, 2009.

PERSIANI, L.; RODA, E.; ROVATI, L. C.; LOCATELLI, M.; GIACOVELLI, G.; RODA, A. Glucosamine oral bioavailability and plasma pharmacokinetics after 
increasing doses of crystalline glucosamine sulfate in man. Osteoarthritis and Cartilage, v. 13, n. 12, p. 1041-1049, dec. 2005. Disponível em: <http://www.sciencedirect. com/science/article/pii/S1063458405001937>. Acesso em: 23 set. 2010.

POND, M.; NUKI, G. Experimentally-induced osteoarthritis in the dog. Annals of the Rheumatic Diseases, v. 32, n. 4, p. 387-8, jul. 1973. Disponível em: $\quad<$ http://www.ncbi.nlm.nih.gov/entrez/query.fcgi $? \mathrm{cmd}=$ Retrieve $\& \mathrm{db}=$ PubMed\&dopt $=$ Citation\&list_ uids $=4726075>$. Acesso em: 10 jan. 2010.

ROGACHEFSKY, R. A.; DEAN, D. D.; HOWELL, D. S.; ALTMAN, R. D. Treatment of canine osteoarthritis with insulin-like growth factor-1 (IGF-1) and sodium pentosan polysulfate. Osteoarthritis and Cartilage, v. 1, n. 2, p. 105-14, apr. 1993. Disponível em: <http://www. ncbi.nlm.nih.gov/pubmed/8886086>. Acesso em: 23 maio 2010 .

SCOTT, H. Central and peripheral parenteral nutrition. Veterinary Focus, v. 7, n. 3, p. 17-18, 2007.

SCHAWARZ, J. Afecções articulares. In: FOSSUM, T. W. Fundamentos de cirurgia ortopédica e manejo de fraturas, cirurgia de pequenos animais. 3. ed. São Paulo: Malone, 2008. p. 1143-1315.

STEFEY, E. P. Inhalation anesthetics. In: THOMPSON, J. C.; TRANQUILLI, W. J.; BENSON, G. J. Lumb \& jones veterinary anesthesia. 3. ed. Baltimore: Lea \& Febiger, 1996. p. 297-329.
TAKIZAWA, M.; YATABE, T.; OKADA, A.; CHIJIIWA, M.; MOCHIZUKI, S.; GHOSH, P.; OKADA, Y. Calcium pentosan polysulfate directly inhibits enzymatic activity of ADAMTS4 (aggrecanase-1) in osteoarthritic chondrocytes. FEBS Letters, v. 582, n. 19, p. 2945-9, aug. 2008. Disponível em: <http://www.ncbi.nlm.nih. gov/pubmed/18671975>. Acesso em: 13 out. 2010.

TORELLI, S. R.; RAHAL, S. C.; VOLPI, R. S.; YAMASHITA, S.; MAMPRIM, M. J.; CROCCI, A. J. Radiography, computed tomography and magnetic resonance imaging at 0.5 Tesla of mechanically induced osteoarthritis in rabbit knees. Brazilian Journal of Medical and Biological Research, v. 37, n. 4, p. 493-501, apr. 2004. Disponível em: <http://www.ncbi.nlm.nih. gov/pubmed/15064812>. Acesso em: 10 jan. 2010.

VOLPI, N. Oral absorption and bioavailability of ichthyic origin chondroitin sulfate in healthy male volunteers. Osteoarthritis and Cartilage, v. 11, n. 6, p. 433-41, 2003. Disponível em: <http://www.ncbi.nlm.nih. gov/pubmed/12801483>. Acesso em: 10 jan. 2010.

Oral bioavailability of chondroitin sulfate (Condrosulf) and its constituents in healthy male volunteers. Osteoarthritis and Cartilage, v. 10, n. 10, p. 768-77, oct. 2002. Disponível em: <http://www.ncbi. nlm.nih.gov/pubmed/12359162>. Acesso em: 10 jan. 2010. 